Giolitti, G. \& Craveri, R. (1957). J. gen. Microbiol. 17, 649.

\title{
A Method for Isolating Streptomyces
}

\author{
By G. GIOLITTI AND R. CRAVERI \\ Montecatini Antibiotic Research Laboratories, Milan, Italy
}

The isolation of Streptomyces from crowded or contaminated cultures is often a matter of some difficulty. We have devised a simple method which has been used with some success in the case of sporulating cultures. A small piece of medium bearing the organism to be isolated is cut out with a sterile spatula and placed on the bottom of the cotton wool plug of a tube containing a sterile nutrient agar slope. The medium will adhere to the plug, leaving the mycelium on the outward surface. The tube is then tapped gently, so that spores are scattered on the fresh medium. The tube should be held at a very slight angle to ensure even distribution; too low an angle will tend to confine

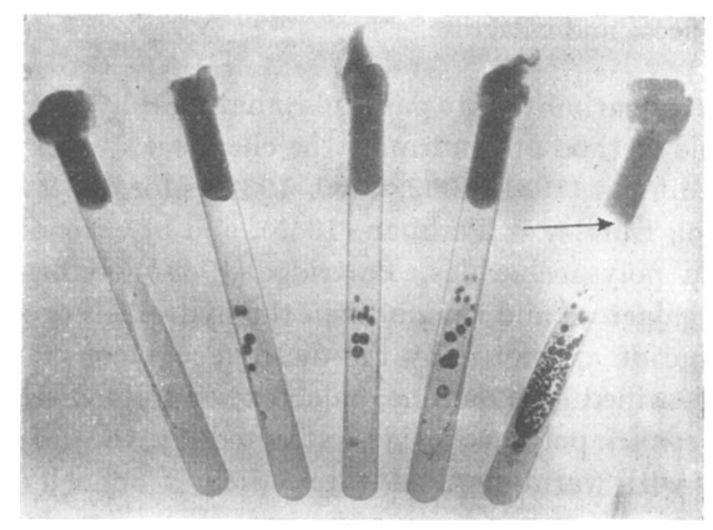

Fig. 1

the spores to the upper portion of the slope. The plug can now be transferred to a second tube and the operation repeated. By this means, a series of tubes bearing decreasing numbers of discrete colonies can be obtained; the last tube may be closed with the original plug. Fig. 1 shows such a series, the position of the inoculum on the plug being indicated by the arrow. In the case of cultures contaminated with bacteria a series of three tubes is usually adequate, since the bacteria remain on the plug, but in the case of fungi or mixed cultures of Streptomyces it is advisable to use a longer series. Apart from the problem of isolation, the method is valuable as a simple way of obtaining isolated colonies on an agar slope culture.

(Received 22 May 1957) 